\title{
Cryo-EM structure of a functional monomeric photosystem I from Thermosynechococcus elongatus reveals red chlorophyll cluster
}

\section{O. Çoruh ${ }^{1,2}$, A. Frank ${ }^{3}$, H. Tanaka ${ }^{1}$, A. Kawamoto' ${ }^{1}$ E. El-Moshnawy ${ }^{4}$, T. Kato ${ }^{5}$, K. Namba ${ }^{6,7,8}$, Christoph Gerle ${ }^{1}$, M.M. Nowaczyk ${ }^{3} \&$ G. Kurisu ${ }^{1,2}$}

\begin{abstract}
${ }^{1}$ Laboratory for Protein Crystallography, Institute for Protein Research, Osaka University, Suita, Osaka, Japan. ${ }^{2}$ Department of Macromolecular Science, Graduate School of Science, Osaka University, Toyonaka, Osaka, Japan. ${ }^{3}$ Plant Biochemistry, Faculty of Biology and Biotechnology, Ruhr-University Bochum, Bochum, Germany. 4 Department of Botany and Microbiology, Faculty of Science, Kafrelsheikh University, Kafr Al Sheikh, Egypt. 5 Laboratory of CryoEM Structural Biology, Institute for Protein Research, Osaka University, Suita, Osaka, Japan. 6 Graduate School of Frontier Biosciences, Osaka University,

Suita, Osaka, Japan. 7 RIKEN Center for Biosystems Dynamics Research and SPring-8 Center, Suita, Osaka, Japan. 8 JEOL

YOKOGUSHI Research Alliance Laboratories, Osaka University, Suita, Osaka, Japan
\end{abstract}

\section{christoph.gerle@riken.jp}

A high-resolution structure of trimeric cyanobacterial Photosystem I (PSI) from Thermosynechococcus elongatus was reported as the first atomic model of PSI almost 20 years ago. However, the monomeric PSI structure has not yet been reported despite long-standing interest in its structure and extensive spectroscopic characterization of the loss of red chlorophylls upon monomerization. Here, we describe the structure of monomeric PSI from Thermosynechococcus elongatus BP-1 as determined by single particle cryo-EM using the CRYO ARM 200 (JEOL). Comparison with the trimer structure gave detailed insights into monomerization-induced changes in both the central trimerization domain and the peripheral regions of the complex. Monomerization-induced loss of red chlorophylls is assigned to a cluster of chlorophylls adjacent to PsaX. Based on our findings, we propose a role of PsaX in the stabilization of red chlorophylls and that lipids of the surrounding membrane present a major source of thermal energy for uphill excitation energy transfer from red chlorophylls to P700.

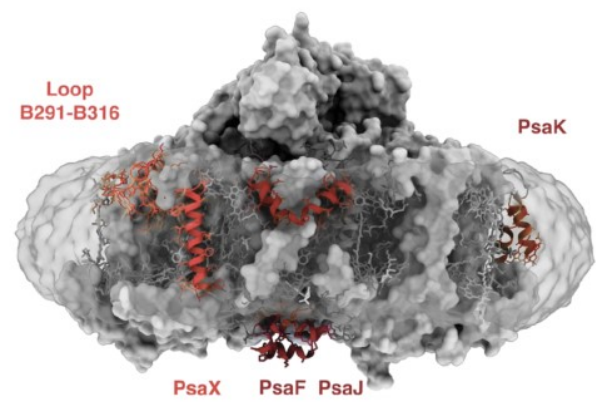

Figure 1. Single particle cryo-EM structure of cyanobacterial monomeric PSI revealing regions of disorder (labled in red) induced by the monomerization of its trimeric form.

[1] Çoruh O, Frank A, Tanaka H, Kawamoto A, El-Mohsnawy E, Kato T, Namba K, Gerle C, Nowaczyk MM, Kurisu G.

(2021). Communications Biology 4-1, 1-16.

[2] Jordan P, Fromme P, Witt HT, Klukas O, Saenger W, Krauß N. (2001). Nature 411, 6840.

[3] Rögner, M., Mühlenhoff, U., Boekema, E. J. \& Witt, H. T. (1990). Biophys. Biochem. Acta Bioenerg. 1015, 415-424.

[4] Pålsson LO, Flemming C, Gobets B, van Grondelle R, Dekker JP, Schlodder E. (1998). Biophys. J. 74, $2611-2622$. 